\section{The Effect of Increased Fiber Ingestion on Lipid Levels and Body Mass: A 4-Week Trial}

\author{
Jeff Golini ${ }^{1}$, Wendy Lou Jones ${ }^{2 *}$ and Ian C Clift ${ }^{3}$ \\ ${ }^{1}$ All American Pharmaceutical and Natural Foods Corporation Billings, \\ Montana, USA \\ ${ }^{2}$ Royal Knight Incorporated, Rochester, Minnesota, USA \\ ${ }^{3}$ Biomedical Associates, Indiana University South Bend, Indiana, USA
}

\begin{abstract}
Hyperlipidemia and hypercholesterolemia are major risk factors for the development of atherosclerosis, coronary artery disease and myocardial infarction. Several studies have suggested that increases in dietary fiber such as those found in plant products may have a positive impact on weight and cholesterol levels-especially when the participant is highly motivated to make personal lifestyle improvements. The current Phase ' 0 ', pilot study, was conducted to assess these effects on a cohort of moderately obese individuals using a proprietary blend of sugar beet pulp, apple pectin, and oat sterols. Although this study found only minor changes in body mass or specific cholesterol markers after 4 weeks, a majority of participants saw a marginal decrease in both total body mass and total cholesterol even when compliance was less than optimal.

Keywords: Dietary fiber; Dietary supplement; High fiber diet; Hyperlipidemia; Obesity
\end{abstract}

\section{Introduction}

Hyperlipidemia and hypercholesterolemia are major risk factors for the development of atherosclerosis, coronary artery disease, and myocardial infarction. Furthermore, studies have shown that dietary fibers play a role in lowering cholesterol in the blood in both humans and animals $[1,2]$. In addition, dietary fibers, such as beta-sitosterol and apple pectin, have been shown to be effective in reducing the size of fat cells through an ability to enhance the functional properties of food [1]. Today, many foods with increased plant sterols, stanols and dietary fiber are used worldwide to reduce serum totals of Low Density Lipoprotein (LDL) cholesterol concentrations by interfering with cholesterol absorption, glucose homeostasis, and thus encouraging weight loss [3-6]. The average fiber intake in the US is less than half of the recommended level, and even lower in those who follow the popular low-carb diets. The addition of dietary fiber to weight loss diets has

*Corresponding author: Wendy Lou Jones, Royal Knight Incorporated, Rochester, USA, Tel: +1 5072898192; E-mail:wlj_1998@yahoo.com

Citation: Golini J, Jones WL, Clift IC (2017) The Effect of Increased Fiber Ingestion on Lipid Levels and Body Mass: A 4-Week Trial. J Food Sci Nut 3: 017.

Received: March 01, 2017; Accepted: April 26, 2017; Published: May 09, 2017 been encouraged to potentially stem the rise in obesity and as a tool to improve success [5].

Sugar-beet pulp (also known as sugar-beet fiber) is a naturally gluten free byproduct of sugar extraction from the sugar-beet. It is made up of predominantly insoluble and soluble fiber [7]. Dietary fiber is defined as a group of non-digestible carbohydrates and lignin which are a part of the intact plant. As such, a fiber-rich diet is also one that is processed and absorbed more slowly [5]. Several studies have suggested that increased satiety and reduced food intake can result from increases in dietary fiber which correlated with weight loss $[5,8,9]$.

Sugar-beet fiber is characterized as containing several of these non-starch polysaccharides. These polysaccharides, which are neither digested nor absorbed by the human small intestine, may be used to remove cholesterol and other substances from the Gastro Intestinal (GI) track, due to its adsorbability and indigestible nature. This material is also used as a component in the production of biogas [10]. It has long been known to be involved in proper bowel function and, more recently in preventing GI symptoms [7]. This may in part be due to the fermentability of sugar-beet pulp by bacteria in the gut, which has been suggested to play a role in cholesterol metabolism $[11,12]$.

Approximately $22 \%$ of sugar-beet pulp is made up of pectin fiber. This material has a confirmed positive impact on cholesterol reduction. It also leads to increased water retention (up to 7-8 times its mass in some instances), causing a rapid increase in the satiety hormones GLP-1 and PYY in rodent models [8], commonly targeted in several medically prescribed diabetes control medications [13]. Diets high in fiber have led to reduced weight in controlled clinical trials [4].

Two other well-known fiber products, apple pectin and oat sterols both have demonstrated important roles in cholesterol lowering as well $[5,6]$.

Sugar-beet pulp is also a source of high hemicelluloses [7,11]. In a porcine study, it was concluded that hemicelluloses were superior to other fiber types in relation to cholesterol reduction [11]. Similar reductions in cholesterol have been noted in farm animals fed sugar beet pulp [14]. Although human studies have been limited, it has been suggested that human gut microorganisms are superior at breaking down the sugar beet pectin oligosaccharides compared to porcine microorganisms [12], which could play a beneficial role in its effectiveness in humans. In a small study conducted by Lampe et al. at the University of Minnesota in 1991, serum cholesterol was reduced more effectively with sugar beet pulp fiber than the control and four other fiber types (cereal or vegetable) given in a loaf of bread each day [15].

All American ${ }^{\circledR}$ Pharmaceutical devised a tableted product that could be consumed as a dietary supplement for use in reducing total cholesterol and increasing the likelihood of a modest weight loss in those individuals motivated to lose weight. To test this product, All American ${ }^{\circledR}$ Pharmaceutical conducted the following four-week, Phase ' 0 ' - Pilot Study to assess the short-term effects of this product on cholesterol, triglycerides, High Density Lipoprotein (HDL) and Low Density Lipoprotein (LDL) involving 12 moderately obese participants; 8 female and 4 males. 


\section{Materials and Methods}

\section{Study site and IRB}

The study was conducted at Montana Medical Research, located at 2683 Palmer Street, Suite B, Missoula, MT 59808. The study received IRB approval under the assigned number PTBP-001/IRB No.: 30365/1. The principal investigator for the study was Dr. Clancy L. Cone.

\section{Participants}

Twelve volunteers, 8 females and 4 males, with moderate obesity, were recruited for this study. These individuals had, at sometime in their past, all attempted dieting with no success. At the time of their recruitment, no one admitted to being on a 'diet', in fact, all participants were significantly beyond the 20 pounds over their ideal weight (according to a body-mass index chart) entrance requirement. No prestudy food assessment was done and there was no attempt at equalizing gender numbers or ages in this Phase ' 0 ' Pilot Study. Only their body mass and cholesterol levels were examined.

\section{Verbal and written instructions to participants}

In this 4 week trial, participants were each additionally asked to 'make an effort to control their food intake' and increase their consumption of dietary fiber through the use of this test product.

\section{Administration}

Participants received written instructions to consume the required number of tablets ( 2 tablets, 3 times daily) between 30 to 45 minutes prior to each meal by chewing them up and swallowing with 8 to 12 ounces of water. These measures were necessary to allow the tablets time to dissolve and rehydrate in their stomachs prior to food consumption. Data was collected on their pre and post study weight, cholesterol, and compliance with tablet ingestion.

\section{Components}

Tablets were manufactured at All American ${ }^{\circledR}$ Pharmaceutical, containing a proprietary mixture of oat plant sterols; $\beta$-extracted from Beta vulgaris (domestic sugar-beet).

\section{Apple pectin}

Pectin is a specific type of dietary fiber. It is a complex polysaccharide present in the plant cell wall and is extracted for commercial purposes from pulp waste for use in gelation, thickening and stabilization of food. Pectin is often used in products such as jams, marmalades, and other textured foods.

\section{Beta-Sitosterol}

Beta-Sitosterol is also a plant derived sterol commonly shown to lower cholesterol and found in many natural food products. It has been considered for its role in cholesterol lowering and potential role as an anti-cancer agent, with studies that have focused on its role in breast cancer, immune health, estrogen production, oxidative stress, glucose metabolism, as well as cardiovascular disease and others.

Apple pectin and beta-sitosterol contribute to cholesterol removal from the GI tract through adsorption.

\section{Sugar-Beet Pulp}

Sugar-beet pulp (also known as sugar-beet fiber) is a natural, gluten free byproduct of sugar extraction from the sugar beet. It is a dietary fiber which is defined as a non-digestible carbohydrate and lignin that are part of the intact plant.

\section{Results and Discussion}

At the completion of the study, a slight majority of the participants; 7 out of 12, saw a decrease in body mass from their initial weights. Overall, only a minimal weight change was noted over the 4-week period; the average starting mass was $111.94 \mathrm{~kg}$ and the average ending mass was $112.30 \mathrm{~kg}$ (Figure 1a). Those with reduced mass saw an average decrease of $0.63 \mathrm{~kg}$ or $1.4 \mathrm{lbs}$ (range: $0.1 \mathrm{~kg}$ to $1.5 \mathrm{~kg}$ ).

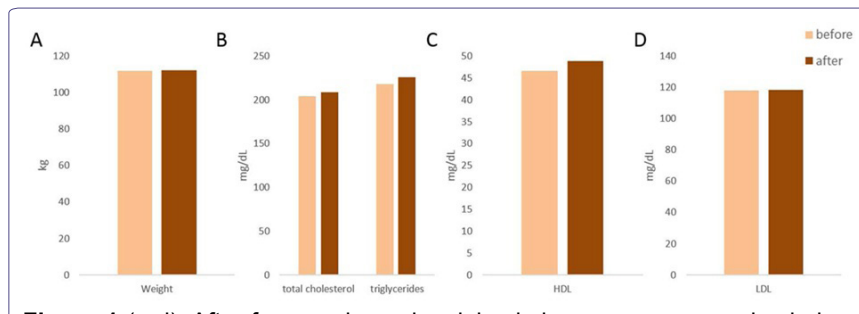

Figure 1 (a-d): After four weeks, only minimal changes were seen in cholesterol and weight.

Total cholesterol increased $4.75 \mathrm{mg} / \mathrm{dL}$ when averaged over all participants, rising from 204 to 208.75 . This was primarily seen in a rise in triglycerides; rising from 218 to $225.58 \mathrm{mg} / \mathrm{dL}$ (Figure $1 \mathrm{~b}$ ), and $\mathrm{HDL}$; rising from 46.58 to $48.83 \mathrm{mg} / \mathrm{dL}$ (Figure 1c). However, total cholesterol decreased in 7 of 12 participants. In this cohort, the range of decrease was 1 to 19 pts with an average of 7.10 pts or $3.52 \%$. Total LDL and total triglycerides also decreased in 6 of the 12 participants with an average decrease of 11.60 pts or $9.58 \%$ and 33.16 pts or $16.65 \%$ respectively. HDL also increased in 7 out of 12 participants. Statistical analysis by paired student $t$-test in this study did not reach significance for any parameter, suggesting that both groups were not discernibly different even after subtracting individuals that were considered less than $90 \%$ compliant. Only the HDL value approached statistical significance with a p-value of 0.14 . When the data was adjusted for over $90 \%$ compliance with study guidelines, only 7 participants remained. In this selected population, even marginal changes in body mass were reduced as were the subtle increases in total cholesterol and triglycerides (Figure 2a-b). However, the subtle increase in HDL remained and a subtle decrease in LDL was noted (Figure 2c-d). This subtle uptick in HDL cholesterol may suggest that long-term ingestion of increased dietary fiber could have a positive effect on the level of good cholesterol, but further studies will be needed. Due to low participant numbers, statistical power was unobtainable. At the end of the study, a survey of participants was conducted to determine their overall viewpoint of the proto-product. Complaints were voiced by all volunteers regarding the taste and texture of the study material.

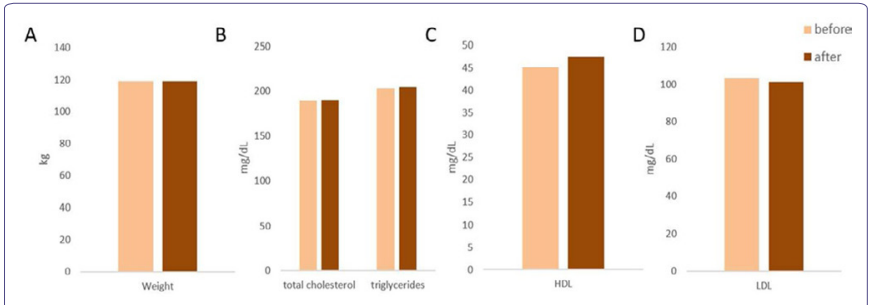

Figure 2 (a-d): A subtle increase in HDL and a subtle decrease in weight and LDL were both noted.

In addition, most participants reported they made no effort to control their diet or change their lifestyle during the study, despite being asked to do so. The experimental period spanned the February holiday, and some participants admitted to 'binging' within days of their 


\begin{tabular}{|c|c|c|c|c|c|c|c|c|c|c|c|c|}
\hline Subject \# & 101 & 102 & 103 & 104 & 105 & 106 & 107 & 108 & 109 & 110 & 111 & 112 \\
\hline Gender & $\mathrm{F}$ & $\mathrm{F}$ & $\mathrm{F}$ & $\mathrm{F}$ & $\mathrm{F}$ & $\mathrm{F}$ & M & M & $\mathrm{F}$ & M & $\mathrm{F}$ & M \\
\hline Compliance(\%) & 74.4 & 95.2 & 96.4 & 100 & 100 & 72.6 & 84.5 & 97.6 & 86.3 & 100 & 96.4 & 83.8 \\
\hline \multicolumn{12}{|c|}{ Start } & \\
\hline Weight(kg) & 94.7 & 116.2 & 188.94 & 106.8 & 92.1 & 113.05 & 88.4 & 125.95 & 83.85 & 110.95 & 90.95 & 131.4 \\
\hline Cholesterol(mg/dL) & 230 & 184 & 174 & 175 & 238 & 249 & 260 & 211 & 258 & 163 & 180 & 126 \\
\hline Triglycerides(mg/dLL) & 146 & 100 & 138 & 304 & 245 & 192 & 514 & 155 & 162 & 225 & 255 & 180 \\
\hline $\mathrm{HDL}(\mathrm{mg} / \mathrm{dL})$ & 67 & 61 & 32 & 37 & 50 & 47 & 42 & 65 & 60 & 46 & 25 & 27 \\
\hline LDL(mg/dL) & 134 & 103 & 114 & 77 & 139 & 164 & 163 & 115 & 166 & 72 & 104 & 63 \\
\hline \multicolumn{12}{|c|}{ End } & \\
\hline Weight(kg) & 95.4 & 116.55 & 192.8 & 105.25 & 92 & 114.35 & 90.9 & 124.4 & 84.2 & 110.75 & 90.1 & 130.9 \\
\hline Cholesterol(mg/dL) & 223 & 165 & 169 & 196 & 230 & 241 & 271 & 209 & 266 & 178 & 181 & 176 \\
\hline Triglycerides(mg/dL) & 160 & 60 & 158 & 255 & 254 & 248 & 465 & 147 & 128 & 206 & 350 & 276 \\
\hline $\mathrm{HDL}(\mathrm{mg} / \mathrm{dL})$ & 62 & 64 & 33 & 44 & 47 & 44 & 47 & 64 & 66 & 56 & 24 & 35 \\
\hline LDL(mg/dL) & 129 & 89 & 104 & 101 & 132 & 147 & 176 & 116 & 174 & 81 & 87 & 86 \\
\hline
\end{tabular}

final blood draw. Overall, the compliance of this study was at or below $90 \%$.

Individual responses can be seen in Table 1.

While no previous studies have been performed with this formulation; a proprietary blend of sugar-beet pulp, in combination with two other fiber sources: apple pectin and oat sterols, similar studies have suggested that the ingestion of a variety of fiber sources, including those combined in this study, may have a beneficial effect on weight control and lipid regulation.

\section{Conclusion}

This pilot study focused on a cohort of moderately obese and mostly less than $100 \%$ compliant individuals. The study results found no major changes in body mass or carbohydrate levels after only four weeks, only minimal changes were seen in cholesterol and weight as shown in figure 1 .

Because the purported effect of this product is through binding in the GI, the ingestion of the product was also difficult to time when medications were also being taken. Despite the lack of statistical power, 7 out of 12 participants saw a decrease in body weight and a reduction in total cholesterol. This indicates that a subset of the population may respond to higher fiber in their diets. Further edification into predictive lifestyle and genetic factors, as well as a sufficiently powered study, are necessary to fully examine the efficacy of a high fiber diet.

\section{Acknowledgment}

All American ${ }^{\oplus}$ Pharmaceutical supported the manufacture of the tablets used in this study.

\section{Disclosure Statement}

All American ${ }^{\oplus}$ Pharmaceutical has a financial interest in the proto-product tested.

\section{References}

1. Sang S, Chu YF (2017) Whole grain oats, more than just a fiber: Role of unique phytochemicals. Molecular Nutrition Food Research.

2. Shinozaki K, Okuda M, Sasaki S, Kunitsugu I, Shigeta M (2015) Dietary Fiber Consumption Decreases the Risks of Overweight and Hypercholesterolemia in Japanese Children. Ann Nutr Metab 67: 58-64.
3. Baumgartner S, Mensink RP, Plat J (2016) Effects of a Plant Sterol or Stanol Enriched Mixed Meal on Postprandial Lipid Metabolism in Healthy Subjects. PLoS One 9: 11.

4. Ma Y, Olendzki BC, Wang J, Persuitte GM, Li W, et al. (2015) Single-Component Versus Multicomponent Dietary Goals for the Metabolic Syndrome: A Randomized Trial. Ann Inter Med 162: 248-257.

5. Rebello C, Greenway FL, Dhurandhar NV (2014) Functional foods to promote weight loss and satiety. Curr Opin Clin Nutr Metab Care. Nov 17: 596-604.

6. Brouns F, Theuwissen E, Adam A, Bell M, Berger A, et al. (2012) Cholesterol-lowering properties of different pectin types in mildly hyper-cholesterolemic men and women. Eur J Clin Nutr 66: 591-599.

7. Nordic Sugar, Sugar Beet Fibre-Physiological effects \& Clinical studies, Nordic Sugar, Copenhagen, Denmark.

8. Adam CL, Williams PA, Garden KE, Thomson LM, Ross AW (2015) Dose-dependent effects of a soluble dietary fiber (pectin) on food intake, adiposity, gut hypertrophy and gut satiety hormone secretion in rats. PloS One 10.

9. Trigueros L, Peña S, Ugidos AV, Sayas-Barberá E, Pérez-Álvarez JA, et al. (2013) Food ingredients as anti-obesity agents: a review. Crit Rev Food Sci Nutr 53: 929-42.

10. Zieminski K, Kowalska-Wentel M (2015) Effect of enzymatic pretreatment on anaerobic co-digestion of sugar beet pulp silage and vinasse. Bioresour Technol 180: 274-280.

11. Kreuzer M, Hanneken H, Wittmann M, Gerdemann MM, Machmuller A (2002) Effects of different fiber sources and fat addition on cholesterol and cholesterol-related lipids in blood serum, bile and body tissues of growing pigs. Journal of Animal Physiology and Animal Nutrition 86: 57-73.

12. Leijdekkers AG, Aguirre M, Venema K, Bosch G, Gruppen H, et al. (2014) In vitro fermentability of sugar beet pulp derived oligosaccharides using human and pig fecal inocula. J Agric Food Chem 62: 1079-1087.

13. Soni H (2016) Peptide-based GLP-1/glucagon co-agonists: A double-edged sword to combat diabesity. Medical Hypotheses 95: 5-9.

14. Pettersson D, Razdan A (1993) Effects of increasing levels of sugar-beet pulp in broiler chicken diets on nutrient digestion and serum lipids. The British Journal of Nutrition 70: 127-137.

15. Lampe JW, Slavin JL, Baglien KS, Thompson WO, Duane WC, et al. (1991) Serum lipid and fecal bile acid changes with cereal, vegetable, and sugar-beet fiber feeding. The American Journal of Clinical Nutrition 53: 1235-1241. 